\title{
Explicit Jacobi elliptic exact solutions for nonlinear partial fractional differential equations
}

\section{Khaled A Gepreel}

\section{"Correspondence:}

kagepreel@yahoo.com

Mathematics Department, Faculty

of Science, Zagazig University,

Zagazig, Egypt

Mathematics Department, Faculty

of Science, Taif University, Taif, Saudi Arabia

\begin{abstract}
In this article, we use the fractional complex transformation to convert nonlinear partial fractional differential equations to nonlinear ordinary differential equations. An algebraic method is improved to construct uniformly a series of exact solutions for some nonlinear time-space fractional partial differential equations. We construct successfully a series of some exact solutions including the elliptic doubly periodic solutions with the aid of computerized symbolic computation software package such as Maple or Mathematica. This method is efficient and powerful in solving a wide classes of nonlinear partial fractional differential equations. The Jacobi elliptic doubly periodic solutions are generated by the trigonometric exact solutions and the hyperbolic exact solutions when the modulus $m \rightarrow 0$ and $m \rightarrow 1$, respectively.

Keywords: fractional calculus; fractional complex transformation; modified Riemann-Liouville derivative; Jacobi elliptic functions; nonlinear fractional differential equations
\end{abstract}

\section{Introduction}

Nonlinear partial fractional equations are very effective for the description of many physical phenomena such as rheology, the damping law, diffusion processes, and the nonlinear oscillation of an earthquake can be modeled with fractional derivatives [1,2]. Also many applications of nonlinear partial fractional differential equations can be found in turbulence and fluid dynamics and nonlinear biological systems [1-10]. There are many methods for finding the approximate solutions for nonlinear partial fractional differential equations such as the Adomian decomposition method [5, 6], the variational iteration method [7], the homotopy perturbation method [8,9], and the homotopy analysis method $[3,10,11]$ and so on. No analytical method had been available before 1998 for nonlinear fractional differential equations. Li and He [12] have proposed the fractional complex transformation to convert the nonlinear partial fractional differential equations into ordinary differential equations so that all analytical methods devoted to advanced calculus can be applied to fractional calculus. Recently Zhang and Zhang [13] have introduced a direct method called the sub-equation method to look for the exact solutions for nonlinear partial fractional differential equations. He [14] has extended the exp-function method to fractional partial differential equations in the sense of a modified RiemannLiouville derivative based on the fractional complex transform. There are many methods

C2014 Gepreel; licensee Springer. This is an Open Access article distributed under the terms of the Creative Commons Attribution License (http://creativecommons.org/licenses/by/2.0), which permits unrestricted use, distribution, and reproduction in any medium, provided the original work is properly cited. 
for solving the nonlinear partial fractional differential equations such as those in $[15,16]$. Fan [17], Zayed et al. [18] and Hong and $\mathrm{Lu}[19,20]$ have proposed an algebraic method for nonlinear partial differential equations to obtain a series of exact wave solutions including the soliton, and rational, triangular periodic, Jacobian, and Weierstrass doubly periodic solutions. In this paper, we will improve the extended proposed algebraic method to solve the nonlinear partial fractional differential equations. Also we use the improved extended proposed algebraic method to construct the Jacobi elliptic exact solutions for the following nonlinear time-space partial fractional differential equations:

(i) First, we have the space-time fractional derivative nonlinear Korteweg-de Vries (KdV) equation [21]

$$
\frac{\partial^{\alpha} u}{\partial t^{\alpha}}+a u \frac{\partial^{\beta} u}{\partial x^{\beta}}+\frac{\partial^{3 \beta} u}{\partial x^{3 \beta}}=0, \quad t>0,0<\alpha, \beta \leq 1,
$$

where $a$ is a constant. When $\alpha, \beta \rightarrow 1$, the $\mathrm{KdV}$ equation has been used to describe a wide range of physics phenomena of the evolution and interaction of nonlinear waves. It was derived from the propagation of dispersive shallow water waves and is widely used in fluid dynamics, aerodynamics, and continuum mechanics, and as a model for shock wave formation, solitons, turbulence, boundary layer behavior, mass transport, and the solution representing the water's free surface over a flat bed [22].

(ii) Then we have the space-time fractional derivative nonlinear fractional ZakharovKunzetsov-Benjamin-Bona-Mahony (ZKBBM) equation [23],

$$
\frac{\partial^{\alpha} u}{\partial t^{\alpha}}+\frac{\partial^{\beta} u}{\partial x^{\beta}}-2 a u \frac{\partial^{\beta} u}{\partial x^{\beta}}-b \frac{\partial^{\alpha}}{\partial t^{\alpha}}\left(\frac{\partial^{2 \beta} u}{\partial x^{2 \beta}}\right)=0, \quad t>0,0<\alpha, \beta \leq 1,
$$

where $a, b$ are arbitrary constants. When $\alpha, \beta \rightarrow 1$, this system has been investigated by Benjamin et al. [23] for the first time, as an alternative model to the KdV equation for long waves and it plays an important role in the modeling of nonlinear dispersive systems. The Benjamin-Bona-Mahony equation is applicable to the study of drift waves in a plasma or Rossby waves in rotating fluids.

\section{Preliminaries}

There are many types of the fractional derivatives such as the Kolwankar-Gangal local fractional derivative [24], Chen's fractal derivative [25], Cresson's derivative [26], and Jumarie's modified Riemann-Liouville derivative [27, 28].

In this section, we give some basic definitions of fractional calculus theory which will be used in this work.

Jumarie's modified Riemann-Liouville derivative of order $\alpha$ is defined as $[28,29]$

$$
D_{x}^{\alpha} f(x)=\frac{1}{\Gamma(1-\alpha)} \frac{d}{d x} \int_{0}^{x}(x-\xi)^{-\alpha}(f(\xi)-f(0)) d \xi, \quad 0<\alpha<1,
$$

where $f: R \rightarrow R, x \mapsto f(x)$ denotes a continuous (but not necessarily first order differentiable) function.

Also the inverse of Jumarie's modified Riemann-Liouville derivative to $f(x)$ of order $\alpha$ in the interval $[a, b]$ is defined by

$$
I_{x}^{\alpha} f(x)=\frac{1}{\Gamma(\alpha)} \int_{0}^{x}(x-\xi)^{\alpha-1} f(\xi) d \xi=\frac{1}{\Gamma(\alpha+1)} \int_{0}^{x} f(\xi)(d x)^{\alpha}, \quad 0<\alpha \leq 1 .
$$


Some properties for the proposed Jumarie's modified Riemann-Liouville derivative are listed from [27-30] as follows:

$$
\begin{aligned}
& \begin{array}{l}
f^{(\alpha)}[x(t)]=\frac{d f}{d x} x^{(\alpha)}(t), \\
D_{x}^{\alpha} x^{\beta}=\frac{1}{\Gamma(1-\alpha)} \frac{d}{d x} \int_{0}^{x} \xi^{\beta}(x-\xi)^{-\alpha} d \xi=\frac{B(1+\beta, 1-\alpha)}{\Gamma(1-\alpha)} \frac{d}{d x}\left(x^{\beta-\alpha+1}\right) \\
\quad=\frac{\Gamma(1+\beta)}{\Gamma(\beta-\alpha+2)} \frac{d}{d x}\left(x^{\beta-\alpha+1}\right)=\frac{\Gamma(1+\beta)}{\Gamma(\beta-\alpha+1)} x^{\beta-\alpha}, \quad \beta>\alpha-1, \\
D_{x}^{\alpha}(f(x) \cdot g(x))=g(x) D_{x}^{\alpha}(f(x))+f(x) D_{x}^{\alpha}(g(x)),
\end{array}
\end{aligned}
$$

where $B$ is the beta function. The function $f(x)$ should be differentiable with respect to $x(t)$ and $x(t)$ is fractional differentiable in (6). The above results are employed in the following sections. The Leibniz rule is given (7) for modified Riemann-Liouville derivative which is modified by Wu in [30].

The modified Riemann-Liouville derivative has been successfully applied in probability calculus [31], fractional Laplace problems [32], the fractional variational approach with several variables [33], the fractional variational iteration method [34], the fractional variational approach with natural boundary conditions [35], and the fractional Lie group method [36].

\section{The improved extended proposed algebraic method for nonlinear partial fractional differential equations}

Consider the following nonlinear partial fractional differential equation:

$$
\begin{aligned}
& F\left(u, D_{t}^{\alpha} u, D_{x}^{\beta} u, D_{y}^{\gamma} u, D_{z}^{\delta} u, D_{t}^{\alpha} D_{t}^{\alpha} u, D_{t}^{\alpha} D_{x}^{\beta} u, D_{x}^{\beta} D_{x}^{\beta} u, D_{x}^{\beta} D_{y}^{\gamma}, D_{y}^{\gamma} D_{y}^{\gamma} u, \ldots\right)=0, \\
& \quad 0<\alpha, \beta, \delta, \gamma \leq 1,
\end{aligned}
$$

where $u$ is an unknown function, $F$ is a polynomial in $u$ and its partial fractional derivatives in which the highest order fractional derivatives and the nonlinear terms are involved.

We give the main steps of the modified extended proposed algebraic method for nonlinear partial fractional differential equations.

Step 1. The fractional complex transformation

$$
u(x, y, z, t)=u(\xi), \quad \xi=\frac{K x^{\beta}}{\Gamma(\beta+1)}+\frac{N y^{\gamma}}{\Gamma(\gamma+1)}+\frac{M z^{\delta}}{\Gamma(\delta+1)}+\frac{L t^{\alpha}}{\Gamma(\alpha+1)},
$$

where $K, L, M$, and $N$ are nonzero arbitrary constants, permits us to reduce (8) to a nonlinear ODE for $u=u(\xi)$ in the following form:

$$
P\left(u, u^{\prime}, u^{\prime \prime}, u^{\prime \prime \prime}, \ldots\right)=0
$$

If possible, we should integrate (10) term by term one or more times.

Step 2. Suppose the solutions of (10) can be expressed by a polynomial in $\phi$ in the form $[17,18]$ :

$$
u(\xi)=\sum_{i=-m}^{m} \alpha_{i} \phi^{i}(\xi), \quad \alpha_{m} \neq 0,
$$


Table 1 The exact solutions of the Jacobi elliptic differential equation (12) when $e_{0}, e_{1}$ and $e_{2}$ take special values

\begin{tabular}{llll}
\hline $\mathbf{e}_{\mathbf{0}}$ & $\boldsymbol{e}_{\mathbf{1}}$ & $\boldsymbol{e}_{\mathbf{2}}$ & $\boldsymbol{\phi}(\boldsymbol{\xi})$ \\
\hline 1 & $-\left(1+m^{2}\right)$ & $m^{2}$ & $\operatorname{sn}(\xi) \operatorname{or} c d(\xi)$ \\
$1-m^{2}$ & $2 m^{2}-1$ & $-m^{2}$ & $c n(\xi)$ \\
$m^{2}-1$ & $2-m^{2}$ & -1 & $d n(\xi)$ \\
$m^{2}$ & $-\left(m^{2}+1\right)$ & 1 & $n s(\xi) \operatorname{or} d c(\xi)$ \\
$-m^{2}$ & $2 m^{2}-1$ & $1-m^{2}$ & $n c(\xi)$ \\
-1 & $2-m^{2}$ & $m^{2}-1$ & $n d(\xi)$ \\
$1-m^{2}$ & $2-m^{2}$ & 1 & $c s(\xi)$ \\
1 & $2-m^{2}$ & $1-m^{2}$ & $s c(\xi)$ \\
1 & $2 m^{2}-1$ & $m^{2}\left(m^{2}-1\right)$ & $s d(\xi)$ \\
$m^{2}\left(m^{2}-1\right)$ & $2 m^{2}-1$ & 1 & $d s(\xi)$ \\
$\frac{1}{4}$ & $\frac{1}{2}\left(1-2 m^{2}\right)$ & $\frac{1}{4}$ & $n s(\xi) \pm c s(\xi)$ \\
$\frac{1}{4}\left(1-m^{2}\right)$ & $\frac{1}{2}\left(1+m^{2}\right)$ & $\frac{1}{4}\left(1-m^{2}\right)$ & $n c(\xi) \pm s c(\xi)$ \\
$\frac{m^{2}}{4}$ & $\frac{1}{2}\left(m^{2}-2\right)$ & $\frac{m^{2}}{4}$ & $\operatorname{sn}(\xi) \pm i c n(\xi)$ \\
$\frac{m^{2}}{4}$ & $\frac{1}{2}\left(m^{2}-2\right)$ & $\frac{1}{4}$ & $n s(\xi) \pm d s(\xi)$ \\
$\frac{m^{2}}{4}$ & $\frac{1}{2}\left(m^{2}-2\right)$ & $\frac{m^{2}}{4}$ & $\sqrt{1-m^{2}} s d(\xi) \pm c d(\xi)$ \\
$\frac{1}{4}$ & $\frac{1}{2}\left(1-m^{2}\right)$ & $\frac{1}{4}$ & $m c d(\xi) \pm i \sqrt{1-m^{2}} n d(\xi)$ \\
$\frac{1}{4}$ & $\frac{1}{2}\left(1-2 m^{2}\right)$ & $\frac{1}{4}$ & $m s n(\xi) \pm i d n(\xi)$ \\
$\frac{1}{4}$ & $\frac{1}{2}\left(1-m^{2}\right)$ & $\frac{1}{4}$ & $\sqrt{1-m^{2}} s c(\xi) \pm d c(\xi)$ \\
$\frac{1}{4}\left(m^{2}-1\right)$ & $\frac{1}{2}\left(1+m^{2}\right)$ & $\frac{1}{4}\left(m^{2}-1\right)$ & $m s d(\xi) \pm n d(\xi)$ \\
$\frac{1}{4}$ & $\frac{1}{2}\left(m^{2}-2\right)$ & $\frac{m^{2}}{4}$ & $\operatorname{sn}(\xi) /(1 \pm d n(\xi))$ \\
\hline
\end{tabular}

where $\alpha_{i}(i=0, \pm 1, \ldots, \pm m)$ are arbitrary constants to be determined later, while $\phi(\xi)$ satisfies the following nonlinear first order Jacobi elliptic differential equation:

$$
\left[\phi^{\prime}(\xi)\right]^{2}=e_{0}+e_{1} \phi^{2}(\xi)+e_{2} \phi^{4}(\xi)
$$

where $e_{0}, e_{1}$, and $e_{2}$ are arbitrary constants.

Step 3 . The positive integer ' $m$ ' can be determined by considering the homogeneous balance between the highest order derivatives and the nonlinear terms appearing in Eq. (10).

Step 4. We must substitute (11) into (10) and using (12), collect all terms with the same order of $\phi(\xi)$ together, then equating each coefficient of the resulting polynomial to be zero. This yields a set of algebraic equations for $\alpha_{i}(i=0, \pm 1, \ldots, \pm m), e_{0}, e_{1}, e_{2}, K, L, M$, and $N$. We then solve this system of algebraic equation with the help of Maple software package to determine $\alpha_{i}(i=0, \pm 1, \ldots, \pm m), e_{0}, e_{1}, e_{2}, K, L, M$, and $N$.

Step 5. The general solutions of (12) have been discussed in $[37,38]$. We put some of the general solutions of (12) from [37] in Table 1.

There are other cases which are omitted here for convenience; see [37].

Step 6. Since the general solutions of (12) are discussed in Table 1, then substituting $\alpha_{i}$ ( $i=0, \pm 1, \ldots, \pm m), e_{0}, e_{1}, e_{2}, K, L, M$, and $N$ and the general solutions of (12) into (11), we obtain more new Jacobi elliptic exact solutions for the nonlinear partial fractional derivative equation (8).

\section{Applications}

In this section, we construct some new Jacobi elliptic exact solutions of some nonlinear partial fractional differential equations via the time-space fractional nonlinear $\mathrm{KdV}$ equation and the time-space fractional nonlinear Zakharov-Kunzetsov-Benjamin-BonaMahomy equation using the modified extended proposed algebraic method which has been paid attention to by many authors. 


\subsection{Example 1: Jacobi elliptic solutions for nonlinear fractional $\mathrm{KdV}$ equation}

In this section, to demonstrate the effectiveness of this method, we use the complex transformation (9) to converting the nonlinear $\mathrm{KdV}$ equation with time-space fractional derivatives (1) to an ordinary differential equation; and we integrate twice, to find

$$
\frac{1}{2} L U^{2}+\frac{a}{6} K U^{3}+\frac{K^{3}}{2}\left[U^{\prime}\right]^{2}+C_{1} U+C_{2}=0,
$$

where $C_{1}$ and $C_{2}$ are the integration constants. Considering the homogeneous balance between the highest order derivatives and the nonlinear terms in (13), we get

$$
U(\xi)=\alpha_{0}+\alpha_{1} \phi(\xi)+\alpha_{2} \phi^{2}(\xi)+\frac{\alpha_{3}}{\phi(\xi)}+\frac{\alpha_{4}}{\phi^{2}(\xi)},
$$

where $\alpha_{0}, \alpha_{1}, \alpha_{2}, a_{3}, a_{4}, L$, and $K$ are arbitrary constants to be determined later. Substituting (14) and (12) into (13), collecting all terms of $\phi(\xi)$, and then setting each coefficient $\phi(\xi)$ to be zero, we get a system of algebraic equations. With the aid of Maple or Mathematica we can solve this system of algebraic equations to obtain the following cases of solutions:

Case 1.

$$
\begin{aligned}
& a_{0}=\frac{2 C_{1}\left(L+4 K^{3} e_{1}\right)}{192 K^{6} e_{0} e_{2}-L^{2}+16 K^{6} e_{1}^{2}}, \quad a_{2}=\frac{24 C_{1} K^{3} e_{2}}{192 K^{6} e_{0} e_{2}-L^{2}+16 K^{6} e_{1}^{2}}, \\
& a=-\frac{192 K^{6} e_{0} e_{2}-L^{2}+16 K^{6} e_{1}^{2}}{4 K C_{1}}, \quad a_{4}=\frac{24 C_{1} K^{3} e_{0}}{192 K^{6} e_{0} e_{2}-L^{2}+16 K^{6} e_{1}^{2}}, \\
& C_{2}=\frac{2 C_{1}^{2}\left(-576 L e_{2} K^{4} e_{0}+4,608 K^{9} e_{0} e_{1} e_{2}+L^{3}-48 K^{6} e_{1}^{2} L-128 K^{9} e_{1}^{3}\right)}{3\left(192 K^{6} e_{0} e_{2}-L^{2}+16 K^{6} e_{1}^{2}\right)^{2}}, \\
& a_{1}=a_{3}=0,
\end{aligned}
$$

where $C_{1}, L, K, e_{0}, e_{1}$, and $e_{2}$ are arbitrary constants.

Case 2.

$$
\begin{aligned}
C_{1}= & \frac{1}{2}\left(192 K^{6} e_{0} e_{2}-L^{2}+16 K^{6} e_{1}^{2}\right) \\
& \times \sqrt{\frac{6 C_{2}}{-576 L e_{2} K^{6} e_{0}+4,608 K^{9} e_{2} e_{1} e_{0}+L^{3}-48 K^{6} e_{1}^{2} L-128 K^{9} e_{1}^{3}}}, \\
a_{0}= & \left(L+4 K^{3} e_{1}\right) \sqrt{\frac{6 C_{2}}{-576 L e_{2} K^{6} e_{0}+4,608 K^{9} e_{2} e_{1} e_{0}+L^{3}-48 K^{6} e_{1}^{2} L-128 K^{9} e_{1}^{3}}}, \\
a_{2}= & 12 K^{3} e_{2} \sqrt{\frac{6 C_{2}}{-576 L e_{2} K^{6} e_{0}+4,608 K^{9} e_{2} e_{1} e_{0}+L^{3}-48 K^{6} e_{1}^{2} L-128 K^{9} e_{1}^{3}}}, \\
a= & -\frac{1}{12 K \sqrt{C_{2}}} \sqrt{6\left[-576 L e_{2} K^{6} e_{0}+4,608 K^{9} e_{2} e_{1} e_{0}+L^{3}-48 K^{6} e_{1}^{2} L-128 K^{9} e_{1}^{3}\right]}, \\
a_{4}= & 12 K^{3} e_{0} \sqrt{\frac{6 C_{2}}{-576 L e_{2} K^{6} e_{0}+4,608 K^{9} e_{2} e_{1} e_{0}+L^{3}-48 K^{6} e_{1}^{2} L-128 K^{9} e_{1}^{3}}}, \\
a_{1}= & a_{3}=0,
\end{aligned}
$$

where $e_{0}, e_{1}, e_{2}, K, L$, and $C_{2}$ are arbitrary constants. 
Note that there are other cases which are omitted here for convenience. Since the solutions obtained here are so many, we just list some of the Jacobi exact solutions corresponding to Case 1 to illustrate the effectiveness of the proposed method. Substituting (15) into (14) we have

$$
\begin{aligned}
u= & \frac{2 C_{1}\left(L+4 K^{3} e_{1}\right)}{192 K^{6} e_{0} e_{2}-L^{2}+16 K^{6} e_{1}^{2}}+\frac{24 C_{1} K^{3} e_{2}}{192 K^{6} e_{0} e_{2}-L^{2}+16 K^{6} e_{1}^{2}} \phi^{2}(\xi) \\
& +\frac{24 C_{1} K^{3} e_{0}}{\left[192 K^{6} e_{0} e_{2}-L^{2}+16 K^{6} e_{1}^{2}\right] \phi^{2}(\xi)}
\end{aligned}
$$

where

$$
\xi=\frac{K x^{\beta}}{\Gamma(\beta+1)}+\frac{L t^{\alpha}}{\Gamma(\alpha+1)} .
$$

According to the general solutions of (12) which are discussed in Table 1, we have the following families of exact solutions:

Family 1. If $e_{0}=1, e_{1}=-\left(1+m^{2}\right), e_{2}=m^{2}$ the exact traveling wave solution takes the form

$$
\begin{aligned}
u_{1}= & \frac{2 C_{1}\left[L-4 K^{3}\left(1+m^{2}\right)\right]}{192 K^{6} m^{2}-L^{2}+16 K^{6}\left(1+m^{2}\right)^{2}} \\
& +\frac{24 C_{1} K^{3} m^{2}}{192 K^{6} m^{2}-L^{2}+16 K^{6}\left(1+m^{2}\right)^{2}} s n^{2}\left[\frac{K x^{\beta}}{\Gamma(\beta+1)}+\frac{L t^{\alpha}}{\Gamma(\alpha+1)}\right] \\
& +\frac{24 C_{1} K^{3}}{\left[192 K^{6} m^{2}-L^{2}+16 K^{6}\left(1+m^{2}\right)^{2}\right]} n s^{2}\left[\frac{K x^{\beta}}{\Gamma(\beta+1)}+\frac{L t^{\alpha}}{\Gamma(\alpha+1)}\right] .
\end{aligned}
$$

To illustrate the behavior of the Jacobi elliptic solution $u_{1}(18)$, see Figure 1 .

Furthermore,

$$
\begin{aligned}
u_{2}= & \frac{2 C_{1}\left[L-4 K^{3}\left(1+m^{2}\right)\right]}{192 K^{6} m^{2}-L^{2}+16 K^{6}\left(1+m^{2}\right)^{2}} \\
& +\frac{24 C_{1} K^{3} m^{2}}{192 K^{6} m^{2}-L^{2}+16 K^{6}\left(1+m^{2}\right)^{2}} c d^{2}\left[\frac{K x^{\beta}}{\Gamma(\beta+1)}+\frac{L t^{\alpha}}{\Gamma(\alpha+1)}\right] \\
& +\frac{24 C_{1} K^{3}}{\left[192 K^{6} m^{2}-L^{2}+16 K^{6}\left(1+m^{2}\right)^{2}\right]} d c^{2}\left[\frac{K x^{\beta}}{\Gamma(\beta+1)}+\frac{L t^{\alpha}}{\Gamma(\alpha+1)}\right],
\end{aligned}
$$
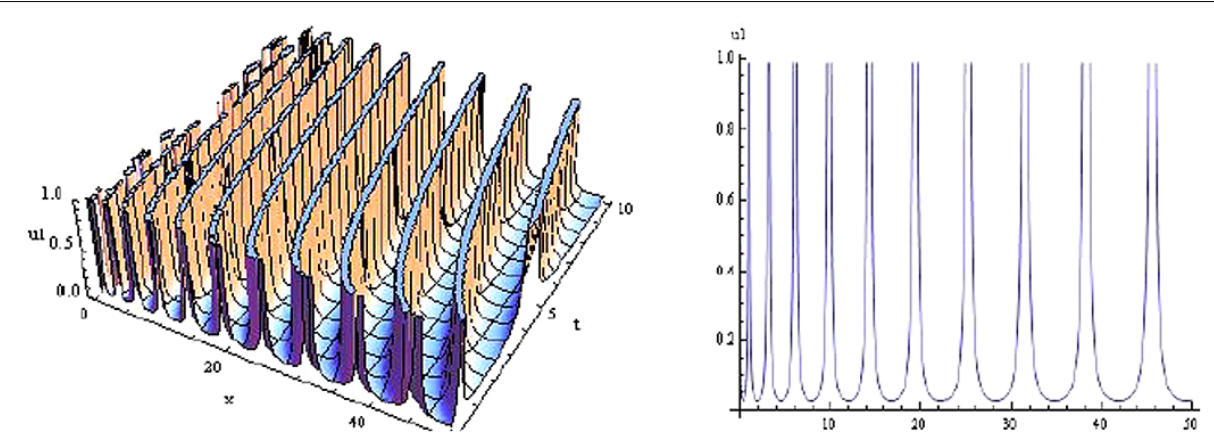

Figure 1 The Jacobi elliptic doubly periodic solution $u_{1}(18)$ and its position at $t=0$, when the parameters $C_{1}=1, L=2, K=3, m=0.2, \alpha=0.5$, and $\beta=0.6$. 


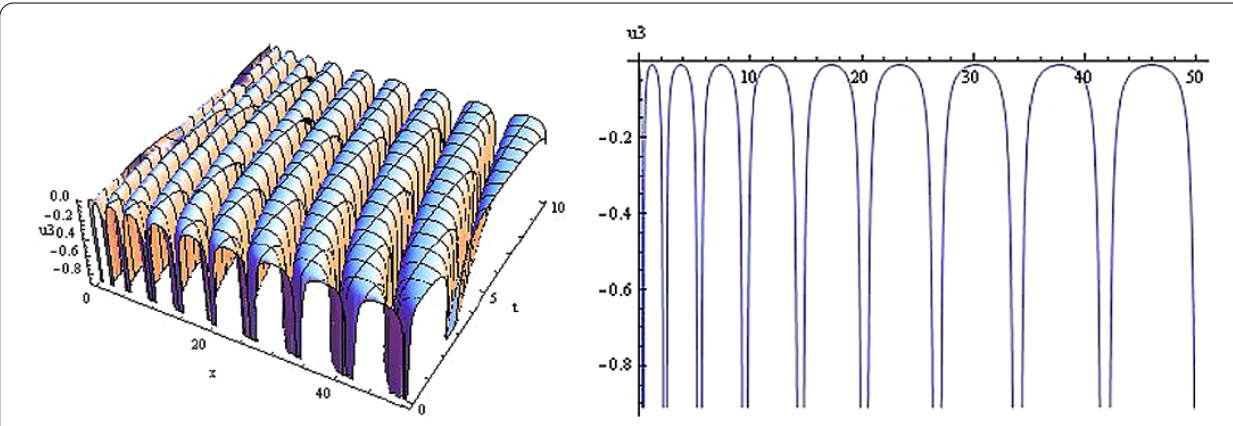

Figure 2 The Jacobi elliptic doubly periodic solution $u_{3}(20)$ and its position at $t=0$, when the parameters $C_{1}=1, L=2, K=3, m=0.5, \alpha=0.5$, and $\beta=0.6$.

where $C_{2}=\frac{2 C_{1}^{2}\left[-576 L m^{2} K^{4}-4,608 K^{9} m^{2}\left(1+m^{2}\right)+L^{3}-48 K^{6}\left(1+m^{2}\right)^{2} L+128 K^{9}\left(1+m^{2}\right)^{3}\right]}{3\left[192 K^{6} m^{2}-L^{2}+16 K^{6}\left(1+m^{2}\right)^{2}\right]^{2}}$.

Family 2. If $e_{0}=1-m^{2}, e_{1}=2 m^{2}-1, e_{2}=-m^{2}$, the exact traveling wave solution takes the form

$$
\begin{aligned}
u_{3}= & \frac{2 C_{1}\left(L+4 K^{3}\left(2 m^{2}-1\right)\right)}{\left\{-192 K^{6}\left(1-m^{2}\right) m^{2}-L^{2}+16 K^{6}\left(2 m^{2}-1\right)^{2}\right\}} \\
& -\frac{24 C_{1} K^{3} m^{2} c n^{2}\left[\frac{K x^{\beta}}{\Gamma(\beta+1)}+\frac{L t^{\alpha}}{\Gamma(\alpha+1)}\right]}{\left\{-192 K^{6}\left(1-m^{2}\right) m^{2}-L^{2}+16 K^{6}\left(2 m^{2}-1\right)^{2}\right\}} \\
& +\frac{24 C_{1} K^{3}\left(1-m^{2}\right) n c^{2}\left[\frac{K x^{\beta}}{\Gamma(\beta+1)}+\frac{L t^{\alpha}}{\Gamma(\alpha+1)}\right]}{\left\{-192 K^{6}\left(1-m^{2}\right) m^{2}-L^{2}+16 K^{6}\left(2 m^{2}-1\right)^{2}\right\}},
\end{aligned}
$$

where $C_{2}=\frac{1}{3\left[-192 K^{6} m^{2}\left(1-m^{2}\right)-L^{2}+16 K^{6}\left(2 m^{2}-1\right)^{2}\right]^{2}}\left\{2 C_{1}^{2}\left(576 L m^{2} K^{4}\left(2 m^{2}-1\right)-4,608 K^{9} m^{2}\left(2 m^{2}-\right.\right.\right.$ 1) $\left.\left.\left(1-m^{2}\right)+L^{3}-48 K^{6}\left(2 m^{2}-1\right)^{2} L-128 K^{9}\left(2 m^{2}-1\right)^{3}\right)\right\}$.

To illustrate the behavior of the Jacobi elliptic solution $u_{3}(20)$, see Figure 2 .

Family 3 . If $e_{0}=m^{2}-1, e_{1}=2-m^{2}, e_{2}=-1$, the exact traveling wave solution takes the form

$$
\begin{aligned}
u_{4}= & \frac{2 C_{1}\left[L+4 K^{3}\left(2-m^{2}\right)\right]}{\left[192 K^{6}\left(1-m^{2}\right)-L^{2}+16 K^{6}\left(2-m^{2}\right)^{2}\right]} \\
& -\frac{24 C_{1} K^{3} d n^{2}\left[\frac{K x^{\beta}}{\Gamma(\beta+1)}+\frac{L t^{\alpha}}{\Gamma(\alpha+1)}\right]}{\left[192 K^{6}\left(1-m^{2}\right)-L^{2}+16 K^{6}\left(2-m^{2}\right)^{2}\right]} \\
& +\frac{24 C_{1} K^{3}\left(m^{2}-1\right) n d^{2}\left[\frac{K x^{\beta}}{\Gamma(\beta+1)}+\frac{L t^{\alpha}}{\Gamma(\alpha+1)}\right]}{\left[192 K^{6}\left(1-m^{2}\right)-L^{2}+16 K^{6}\left(2-m^{2}\right)^{2}\right]},
\end{aligned}
$$

where $C_{2}=\frac{2 C_{1}^{2}\left(576 L K^{4}\left(m^{2}-1\right)-4,608 K^{9}\left(m^{2}-1\right)\left(2-m^{2}\right)+L^{3}-48 K^{6}\left(2-m^{2}\right)^{2} L-128 K^{9}\left(2-m^{2}\right)^{3}\right)}{3\left[192 K^{6}\left(1-m^{2}\right)-L^{2}+16 K^{6}\left(2-m^{2}\right)^{2}\right]^{2}}$.

Family 4. If $e_{0}=\frac{1}{4}\left(1-m^{2}\right), e_{1}=\frac{1}{2}\left(1+m^{2}\right), e_{2}=\frac{1}{4}\left(1-m^{2}\right)$, the exact traveling wave solution takes the form

$$
\begin{aligned}
u_{5}= & \frac{2 C_{1}\left(L+2 K^{3}\left(1+m^{2}\right)\right)}{\left[12 K^{6}\left(1-m^{2}\right)^{2}-L^{2}+4 K^{6}\left(1+m^{2}\right)^{2}\right]} \\
& +\frac{6 C_{1} K^{3}\left(1-m^{2}\right)\left[n c\left(\frac{K x^{\beta}}{\Gamma(\beta+1)}+\frac{L t^{\alpha}}{\Gamma(\alpha+1)}\right) \pm s c\left(\frac{K x^{\beta}}{\Gamma(\beta+1)}+\frac{L t^{\alpha}}{\Gamma(\alpha+1)}\right)\right]^{2}}{\left[12 K^{6}\left(1-m^{2}\right)^{2}-L^{2}+4 K^{6}\left(1+m^{2}\right)^{2}\right]}
\end{aligned}
$$




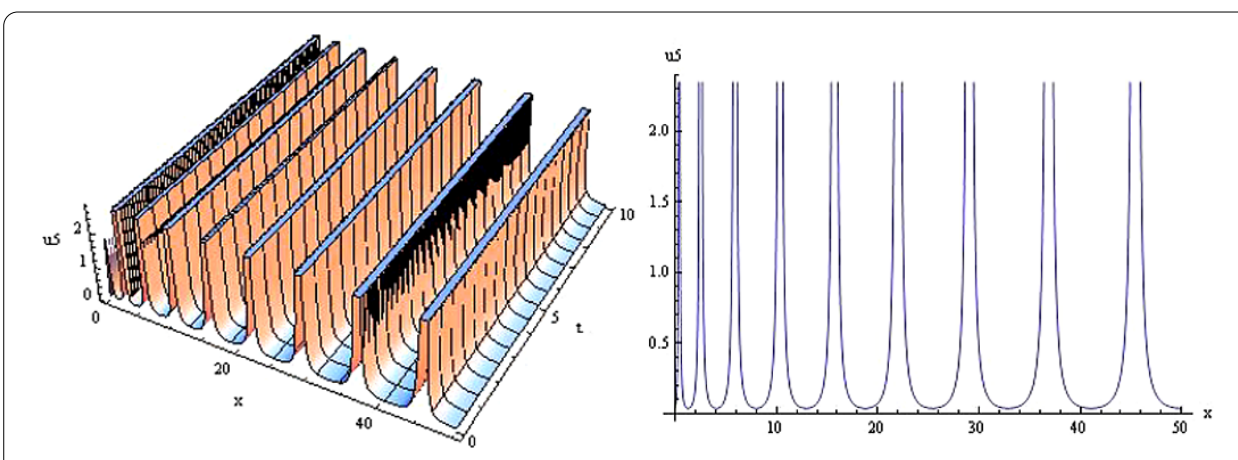

Figure 3 The Jacobi elliptic doubly periodic solution $u_{5}(22)$ and its position at $t=0$, when the parameters $C_{1}=1, L=2, K=3, m=0.5, \alpha=0.5$, and $\beta=0.6$.

$$
\begin{aligned}
& +\frac{6 C_{1} K^{3}\left(1-m^{2}\right)}{\left[12 K^{6}\left(1-m^{2}\right)^{2}-L^{2}+4 K^{6}\left(1+m^{2}\right)^{2}\right]} \\
& \times \frac{1}{\left[n c\left(\frac{K x^{\beta}}{\Gamma(\beta+1)}+\frac{L t^{\alpha}}{\Gamma(\alpha+1)}\right) \pm s c\left(\frac{K x^{\beta}}{\Gamma(\beta+1)}+\frac{L t^{\alpha}}{\Gamma(\alpha+1)}\right)\right]^{2}},
\end{aligned}
$$

where $C_{2}=\frac{2 C_{1}^{2}\left(-36 L K^{4}\left(1-m^{2}\right)^{2}+144 K^{9}\left(1-m^{2}\right)^{2}\left(1+m^{2}\right)+L^{3}-12 K^{6}\left(1+m^{2}\right)^{2} L-16 K^{9}\left(1+m^{2}\right)^{3}\right)}{3\left[12 K^{6}\left(1-m^{2}\right)^{2}-L^{2}+4 K^{6}\left(1+m^{2}\right)^{2}\right]^{2}}$.

To illustrate the behavior of the Jacobi elliptic solution $u_{5}$ (22), see Figure 3.

Family 5. If $e_{0}=\frac{m^{2}}{4}, e_{1}=\frac{1}{2}\left(m^{2}-2\right), e_{2}=\frac{m^{2}}{4}$, the exact traveling wave solution takes the form

$$
\begin{aligned}
u_{6}= & \frac{2 C_{1}\left(L+2 K^{3}\left(m^{2}-2\right)\right)}{12 K^{6} m^{4}-L^{2}+4 K^{6}\left(m^{2}-2\right)^{2}} \\
& +\frac{6 C_{1} K^{3} m^{2}\left[\operatorname{sn}\left(\frac{K x^{\beta}}{\Gamma(\beta+1)}+\frac{L t^{\alpha}}{\Gamma(\alpha+1)}\right) \pm i c n\left(\frac{K x^{\beta}}{\Gamma(\beta+1)}+\frac{L t^{\alpha}}{\Gamma(\alpha+1)}\right)\right]^{2}}{\left[12 K^{6} m^{4}-L^{2}+4 K^{6}\left(m^{2}-2\right)^{2}\right]} \\
& +\frac{24 C_{1} K^{3} m^{2}}{\left[12 K^{6} m^{4}-L^{2}+4 K^{6}\left(m^{2}-2\right)^{2}\right]} \\
& \times \frac{1}{\left[\operatorname{sn}\left(\frac{K x^{\beta}}{\Gamma(\beta+1)}+\frac{L t^{\alpha}}{\Gamma(\alpha+1)}\right) \pm i c n\left(\frac{K x^{\beta}}{\Gamma(\beta+1)}+\frac{L t^{\alpha}}{\Gamma(\alpha+1)}\right)\right]^{2}},
\end{aligned}
$$

where $C_{2}=\frac{2 C_{1}^{2}\left(-36 L K^{4} m^{4}+144 K^{9} m^{4}\left(m^{2}-2\right)+L^{3}-12 K^{6}\left(m^{2}-2\right)^{2} L-16 K^{9}\left(m^{2}-2\right)^{3}\right)}{3\left[12 K^{6} m^{4}-L^{2}+4 K^{6}\left(m^{2}-2\right)^{2}\right]^{2}}$.

To illustrate the behavior of the Jacobi elliptic solution $u_{6}$ (23), see Figures 4 and 5 .

Similarly, we can write down the other families of exact solutions of (1) which are omitted for convenience.

\subsection{Example 2: Jacobi elliptic solutions for nonlinear fractional ZKBBM equation}

In this section we use the proposed method to find the Jacobi elliptic solutions for the nonlinear fractional ZKBBM equation with time and space fractional derivatives (2). The complex fractional transformations (9) convert the nonlinear fractional ZKBBM equation (2) to the following nonlinear ordinary differential equation:

$$
(L+K) U-a K U^{2}-b K^{2} U^{\prime \prime}+C_{1}=0,
$$



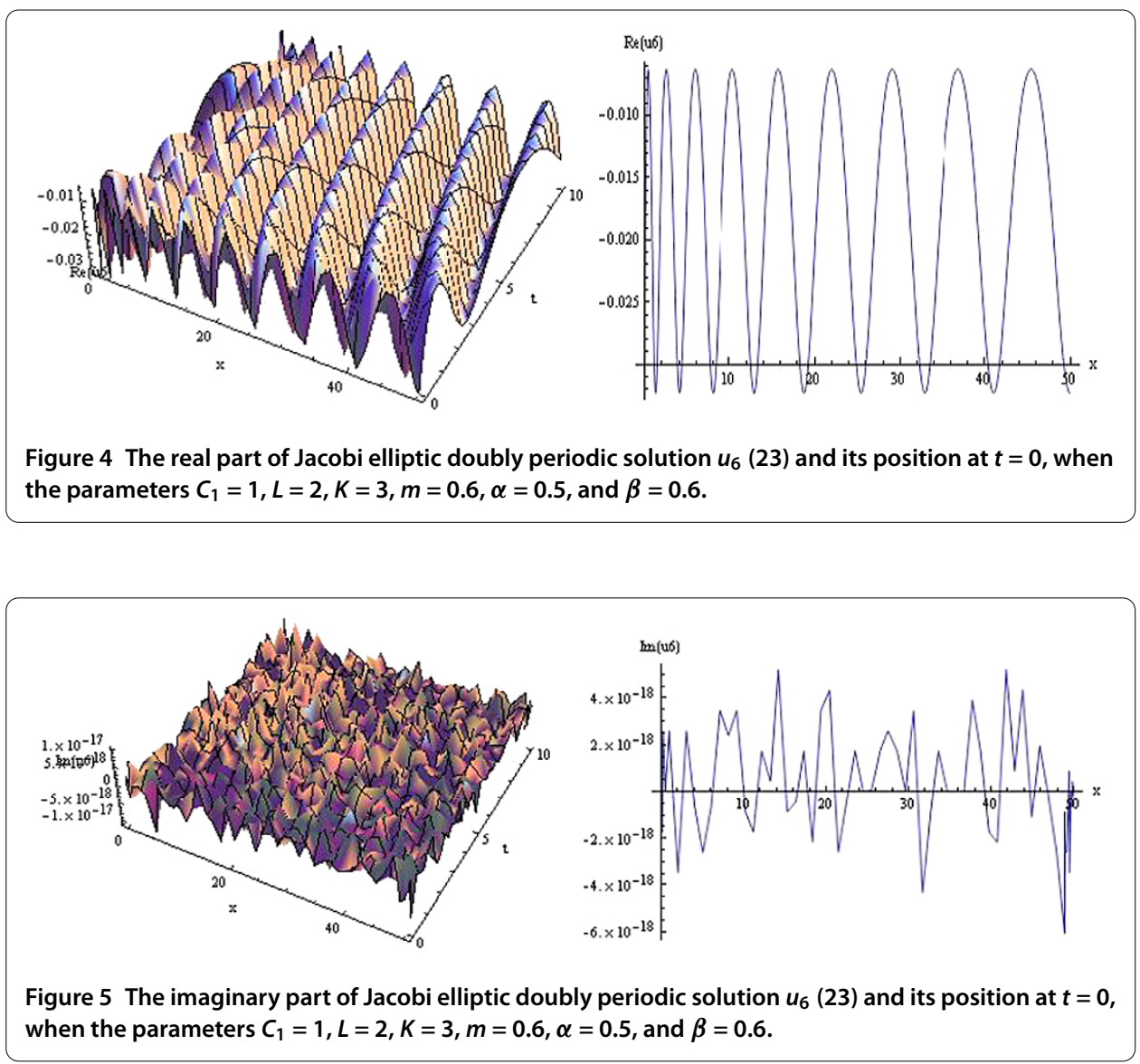

where $C_{1}$ is the integration constant. Considering the homogeneous balance between the highest order derivative and the nonlinear term in (24), we have

$$
U(\xi)=\alpha_{0}+\alpha_{1} \phi(\xi)+\alpha_{2} \phi^{2}(\xi)+\frac{\alpha_{3}}{\phi(\xi)}+\frac{\alpha_{4}}{\phi^{2}(\xi)},
$$

where $\alpha_{0}, \alpha_{1}, \alpha_{2}, a_{3}, a_{4}, L$, and $K$ are arbitrary constants to be determined later. Substituting (25) and (12) into (24), collecting all the terms of powers of $\phi(\xi)$ and setting each coefficient $\phi(\xi)$ to zero, we get a system of algebraic equations. With the aid of Maple or Mathematica we can solve this system of algebraic equations to obtain the following sets of solutions:

Case 1.

$$
\begin{aligned}
& a_{0}=-\frac{4 b K^{2} L e_{1}-K-L}{2 a K}, \quad a_{2}=-\frac{6 K b L e_{2}}{a}, \quad a_{4}=-\frac{6 K b L e_{0}}{a}, \\
& C_{1}=\frac{1}{4 a K}\left\{-K^{2}-2 L K+192 b^{2} K^{4} L^{2} e_{0} e_{2}-L^{2}+16 b^{2} K^{4} L^{2} e_{1}^{2}\right\}, \\
& a_{1}=a_{3}=0
\end{aligned}
$$

where $b, L, K, e_{0}, e_{1}$, and $e_{2}$ are arbitrary nonzero constants. There are many other cases which are omitted for convenience. 
Substituting (26) into (25) we have

$$
u=-\frac{4 b K^{2} L e_{1}-K-L}{2 a K}-\frac{6 K b L e_{2}}{a} \phi^{2}(\xi)-\frac{6 K b L e_{0}}{a \phi^{2}(\xi)}
$$

where

$$
\xi=\frac{K x^{\beta}}{\Gamma(\beta+1)}+\frac{L t^{\alpha}}{\Gamma(\alpha+1)}
$$

and

$$
C_{1}=\frac{1}{4 a K}\left\{-K^{2}-2 L K+192 b^{2} K^{4} L^{2} e_{0} e_{2}-L^{2}+16 b^{2} K^{4} L^{2} e_{1}^{2}\right\}
$$

According to the general solutions of (12) which are discussed in Table 1, we have the following families of Jacobi elliptic exact solutions to the nonlinear ZKBBM equation:

Family 1 . If $e_{0}=1, e_{1}=-\left(1+m^{2}\right), e_{2}=m^{2}$, the exact traveling wave solution takes the form

$$
\begin{aligned}
u_{1}= & \frac{4 b K^{2} L\left(1+m^{2}\right)+K+L}{2 a K}-\frac{6 K b L m^{2}}{a} s n^{2}\left[\frac{K x^{\beta}}{\Gamma(\beta+1)}+\frac{L t^{\alpha}}{\Gamma(\alpha+1)}\right] \\
& -\frac{6 K b L}{a} n s^{2}\left[\frac{K x^{\beta}}{\Gamma(\beta+1)}+\frac{L t^{\alpha}}{\Gamma(\alpha+1)}\right] .
\end{aligned}
$$

To illustrate the behavior of the Jacobi elliptic solution $u_{1}(29)$, see Figure 6.

Furthermore

$$
\begin{aligned}
u_{2}= & \frac{4 b K^{2} L\left(1+m^{2}\right)+K+L}{2 a K}-\frac{6 K b L m^{2}}{a} c d^{2}\left[\frac{K x^{\beta}}{\Gamma(\beta+1)}+\frac{L t^{\alpha}}{\Gamma(\alpha+1)}\right] \\
& -\frac{6 K b L}{a} d c^{2}\left[\frac{K x^{\beta}}{\Gamma(\beta+1)}+\frac{L t^{\alpha}}{\Gamma(\alpha+1)}\right],
\end{aligned}
$$

where $C_{1}=\frac{1}{4 a K}\left\{-K^{2}-2 L K+192 b^{2} K^{4} L^{2} m^{2}-L^{2}+16 b^{2} K^{4} L^{2}\left(1+m^{2}\right)^{2}\right\}$.

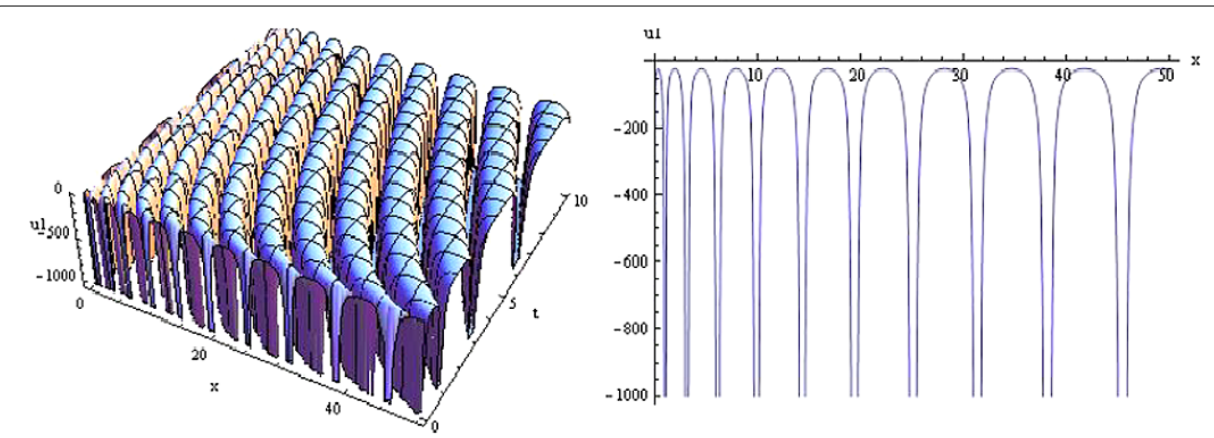

Figure 6 The Jacobi elliptic doubly periodic solution $u_{1}(29)$ and its position at $t=0$, when the parameters $a=1, b=2, L=3, K=4, m=0.2, \alpha=0.5$, and $\beta=0.6$. 


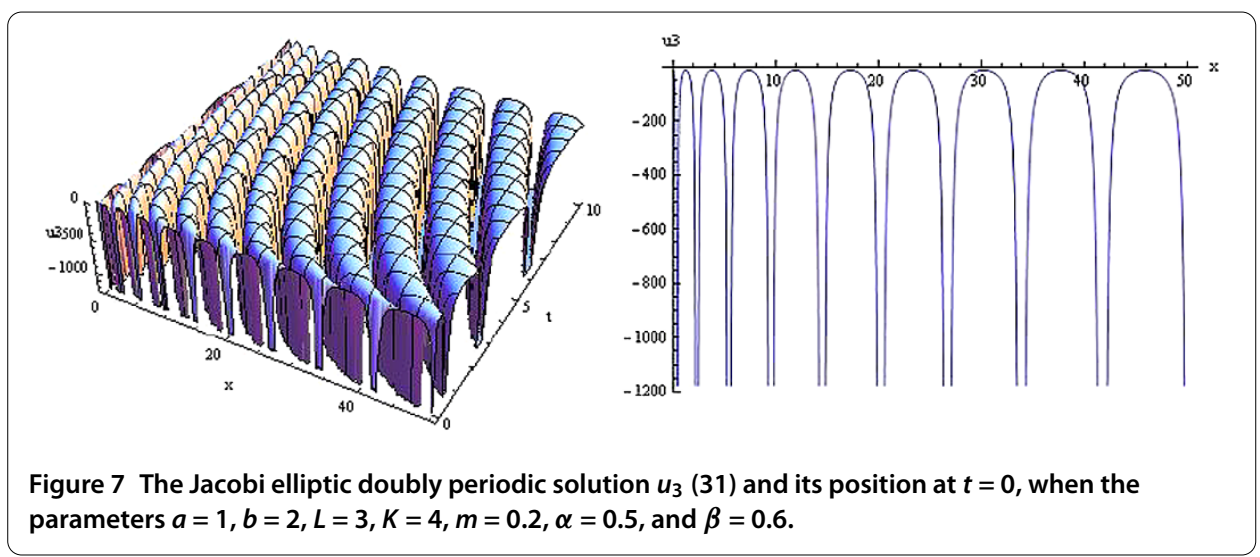

Family 2. If $e_{0}=1-m^{2}, e_{1}=2 m^{2}-1, e_{2}=-m^{2}$, the exact traveling wave solution takes the form

$$
\begin{aligned}
u_{3}= & -\frac{4 b K^{2} L\left(2 m^{2}-1\right)-K-L}{2 a K}+\frac{6 K b L m^{2}}{a} c n^{2}\left(\frac{K x^{\beta}}{\Gamma(\beta+1)}+\frac{L t^{\alpha}}{\Gamma(\alpha+1)}\right) \\
& -\frac{6 K b L\left(1-m^{2}\right)}{a} n c^{2}\left(\frac{K x^{\beta}}{\Gamma(\beta+1)}+\frac{L t^{\alpha}}{\Gamma(\alpha+1)}\right),
\end{aligned}
$$

where $C_{1}=\frac{1}{4 a K}\left\{-K^{2}-2 L K-192 b^{2} K^{4} L^{2}\left(1-m^{2}\right) m^{2}-L^{2}+16 b^{2} K^{4} L^{2}\left(2 m^{2}-1\right)^{2}\right\}$.

To illustrate the behavior of the Jacobi elliptic solution $u_{3}$ (31), see Figure 7 .

Family 3. If $e_{0}=m^{2}-1, e_{1}=2-m^{2}, e_{2}=-1$, the exact traveling wave solution takes the form

$$
\begin{aligned}
u_{4}= & -\frac{4 b K^{2} L\left(2-m^{2}\right)-K-L}{2 a K}+\frac{6 K b L}{a} d n^{2}\left[\frac{K x^{\beta}}{\Gamma(\beta+1)}+\frac{L t^{\alpha}}{\Gamma(\alpha+1)}\right] \\
& -\frac{6 K b L\left(m^{2}-1\right)}{a} n d^{2}\left[\frac{K x^{\beta}}{\Gamma(\beta+1)}+\frac{L t^{\alpha}}{\Gamma(\alpha+1)}\right],
\end{aligned}
$$

where $C_{1}=\frac{1}{4 a K}\left\{-K^{2}-2 L K-192 b^{2} K^{4} L^{2}\left(m^{2}-1\right)-L^{2}+16 b^{2} K^{4} L^{2}\left(2-m^{2}\right)^{2}\right\}$.

Family 4. If $e_{0}=\frac{1}{4}\left(1-m^{2}\right), e_{1}=\frac{1}{2}\left(1+m^{2}\right), e_{2}=\frac{1}{4}\left(1-m^{2}\right)$, the exact traveling wave solution takes the form

$$
\begin{aligned}
u_{5}= & -\frac{2 b K^{2} L\left(1+m^{2}\right)-K-L}{2 a K} \\
& -\frac{3 K b L\left(1-m^{2}\right)}{2 a}\left[n c\left(\frac{K x^{\beta}}{\Gamma(\beta+1)}+\frac{L t^{\alpha}}{\Gamma(\alpha+1)}\right) \pm s c\left(\frac{K x^{\beta}}{\Gamma(\beta+1)}+\frac{L t^{\alpha}}{\Gamma(\alpha+1)}\right)\right]^{2} \\
& -\frac{3 K b L\left(1-m^{2}\right)}{2 a\left[n c\left(\frac{K x^{\beta}}{\Gamma(\beta+1)}+\frac{L t^{\alpha}}{\Gamma(\alpha+1)}\right) \pm s c\left(\frac{K x^{\beta}}{\Gamma(\beta+1)}+\frac{L t^{\alpha}}{\Gamma(\alpha+1)}\right)\right]^{2}},
\end{aligned}
$$

where $C_{1}=\frac{1}{4 a K}\left\{-K^{2}-2 L K+12 b^{2} K^{4} L^{2}\left(1-m^{2}\right)^{2}-L^{2}+4 b^{2} K^{4} L^{2}\left(1+m^{2}\right)^{2}\right\}$.

To illustrate the behavior of the Jacobi elliptic solution $u_{5}$ (33), see Figure 8. 

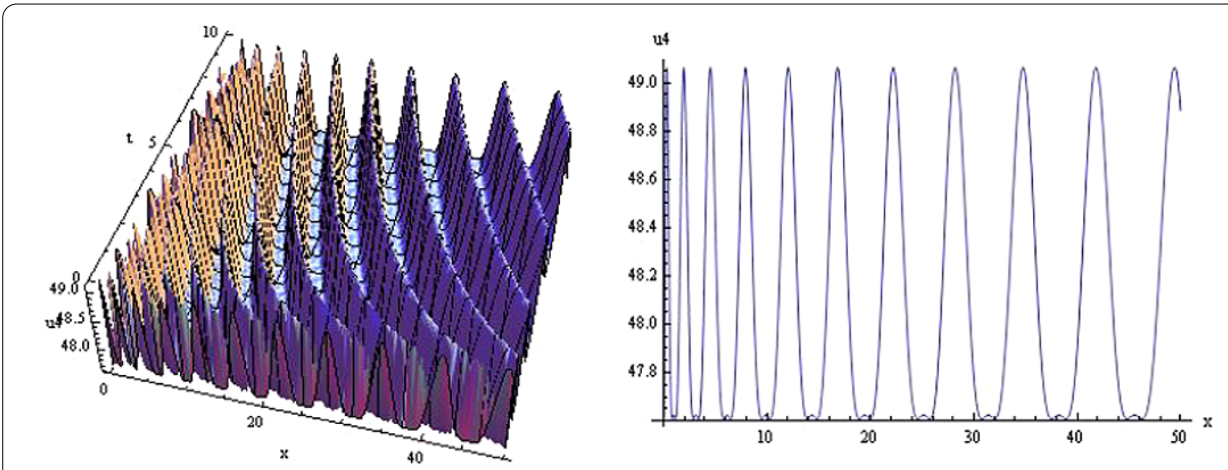

Figure 8 The Jacobi elliptic doubly periodic solution $u_{4}(32)$ and its position at $t=0$, when the parameters $a=1, b=2, L=3, K=4, m=0.2, \alpha=0.5$, and $\beta=0.6$.

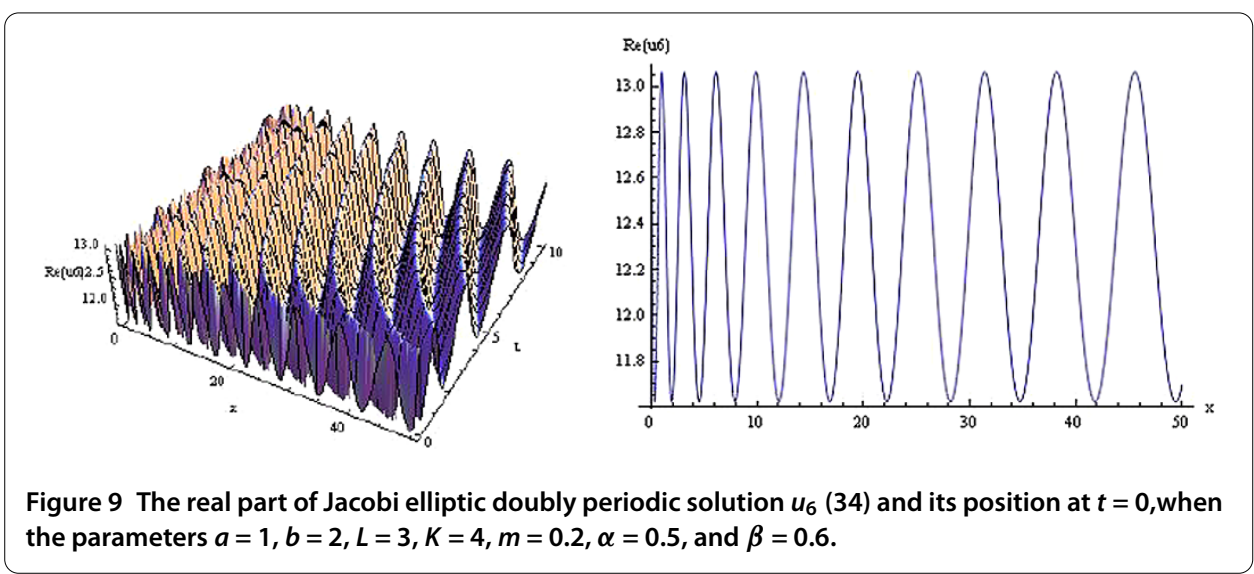

Family 5. If $e_{0}=\frac{m^{2}}{4}, e_{1}=\frac{1}{2}\left(m^{2}-2\right), e_{2}=\frac{m^{2}}{4}$, the exact traveling wave solution takes the form

$$
\begin{aligned}
u_{6}= & -\frac{2 b K^{2} L\left(m^{2}-2\right)-K-L}{2 a K} \\
& -\frac{3 K b L m^{2}}{2 a}\left[\operatorname{sn}\left(\frac{K x^{\beta}}{\Gamma(\beta+1)}+\frac{L t^{\alpha}}{\Gamma(\alpha+1)}\right) \pm i c n\left(\frac{K x^{\beta}}{\Gamma(\beta+1)}+\frac{L t^{\alpha}}{\Gamma(\alpha+1)}\right)\right]^{2} \\
& -\frac{3 K b L m^{2}}{2 a\left[\operatorname{sn}\left(\frac{K x^{\beta}}{\Gamma(\beta+1)}+\frac{L t^{\alpha}}{\Gamma(\alpha+1)}\right) \pm i c n\left(\frac{K x^{\beta}}{\Gamma(\beta+1)}+\frac{L t^{\alpha}}{\Gamma(\alpha+1)}\right)\right]^{2}},
\end{aligned}
$$

where $C_{1}=\frac{1}{4 a K}\left\{-K^{2}-2 L K+12 b^{2} K^{4} L^{2} m^{4}-L^{2}+4 b^{2} K^{4} L^{2}\left(m^{2}-2\right)^{2}\right\}$.

To illustrate the behavior of the Jacobi elliptic solution $u_{6}$ (34), see Figures 9 and 10 .

Similarly, we can write down the other families of exact solutions of (2) which are omitted for convenience.

\section{Conclusion}

In this article we constructed the Jacobi elliptic exact solutions for the nonlinear partial fractional differential equations with the help of the complex fractional transformation and the improved extended proposed algebraic method. This method is effective and powerful for finding the Jacobi elliptic solutions for nonlinear fractional differential equations. 


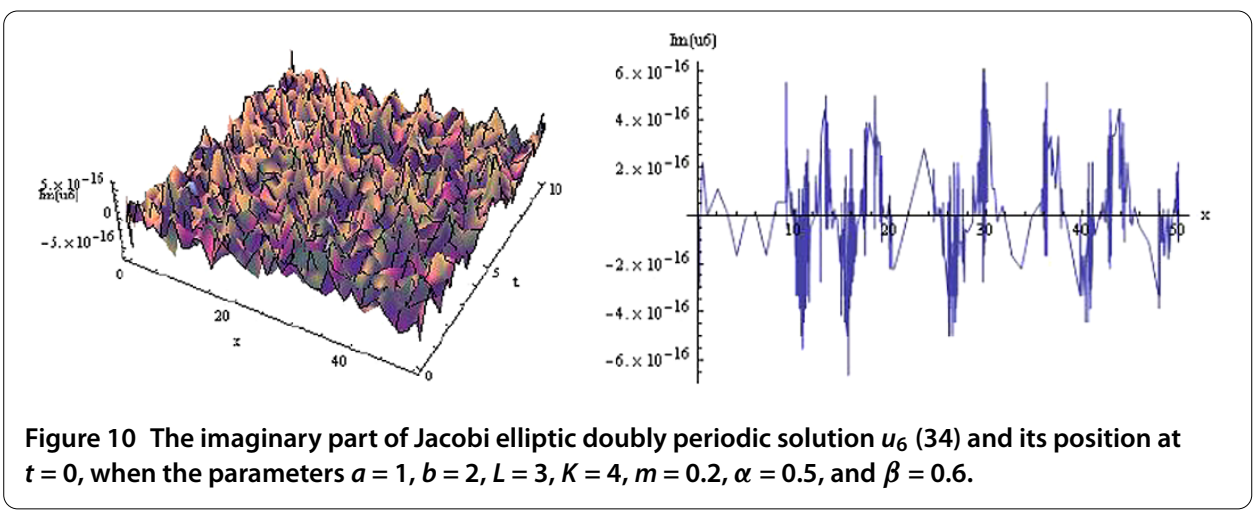

Jacobi elliptic solutions are generalized to the hyperbolic exact solutions and trigonometric exact solutions when the modulus $m \rightarrow 1$ and $m \rightarrow 0$, respectively.

\section{Competing interests}

The author declares to have no competing interests.

\section{Author's contributions}

The author read and approved the final manuscript.

\section{Acknowledgements}

The author would like to thank the editors and the anonymous referees for their careful reading of the paper.

\section{Received: 20 August 2014 Accepted: 24 October 2014 Published: 04 Nov 2014}

\section{References}

1. Podlubny, I: Fractional Differential Equations. Academic Press, San Diego (1999)

2. He, JH: Some applications of nonlinear fractional differential equations and their applications. Bull. Sci. Technol. 15, 86-90 (1999)

3. Mohamed, SM, Al-Malki, F, Talib, R: Approximate analytical and numerical solutions to fractional Newell-Whitehead equation by fractional complex transform. Int. J. Appl. Math. 26, 657-669 (2013)

4. Erturk, VS, Odibat, SM: Application of generalized differential transform method to multi-order fractional differential equations. Commun. Nonlinear Sci. Numer. Simul. 13, 1642-1654 (2008)

5. Daftardar-Gejji, V, Bhalekar, S: Solving multi-term linear and non-linear diffusion wave equations of fractional order by Adomian decomposition method. Appl. Math. Comput. 202, 113-120 (2008)

6. Herzallah, MA, Gepreel, KA: Approximate solution to the time-space fractional cubic nonlinear Schrodinger equation. Appl. Math. Model. 36, 5678-5685 (2012)

7. Sweilam, NH, Khader, MM, Al-Bar, RF: Numerical studies for a multi-order fractional differential equation. Phys. Lett. A 371, 26-33 (2007)

8. Golbabai, A, Sayevand, K: Fractional calculus - a new approach to the analysis of generalized fourth-order diffusion-wave equations. Comput. Math. Appl. 61, 2227-2231 (2011)

9. Gepreel, KA: The homotopy perturbation method to the nonlinear fractional Kolmogorov-Petrovskii-Piskunov equations. Appl. Math. Lett. 24, 1428-1434 (2011)

10. Gepreel, KA, Mohamed, SM: Analytical approximate solution for nonlinear space-time fractional Klein-Gordon equation. Chin. Phys. B 22, 010201 (2013)

11. Mohamed, SM, Al-Malki, F, Talib, R: Jacobi elliptic numerical solutions for the time fractional dispersive long wave equation. Int. J. Pure Appl. Math. 80, 635-646 (2012)

12. $\mathrm{Li}, \mathrm{ZB}, \mathrm{He}, \mathrm{JH}$ : Fractional complex transformation for fractional differential equations. Math. Comput. Appl. 15, 970-973 (2010)

13. Zhang, S, Zhang, HQ: Fractional sub-equation method and its applications to nonlinear fractional PDEs. Phys. Lett. A 375, 1069-1073 (2011)

14. He, JH: Exp-function method for fractional differential equations. Int. J. Nonlinear Sci. Numer. Simul. 13, 363-366 (2013)

15. Gepreel, KA, Al-Thobaiti, AA: Exact solution of nonlinear partial fractional differential equations using the fractional sub-equation method. Indian J. Phys. 88, 293-300 (2014)

16. Bekir, A, Güner, O: Exact solutions of nonlinear fractional differential equations by $\left(G^{\prime} / G\right)$-expansion method. Chin. Phys. B 22, 110202 (2013)

17. Fan, E: Multiple travelling wave solutions of nonlinear evolution equations using a unified algebraic method. J. Phys. A, Math. Gen. 35, 6853-6872 (2002)

18. Zayed, EME, Gepreel, KA, El Horbaty, MM: Extended proposed algorithm with symbolic computation to construct exact solutions for some nonlinear differential equations. Chaos Solitons Fractals 40, 436-452 (2009)

19. Hong, B, Lu, D: New Jacobi elliptic function-like solutions for the general KdV equation with variable coefficients. Math. Comput. Model. 55, 1594-1600 (2012) 
20. Hong, B, Lu, D: New exact solutions for the generalized variable-coefficient Gardner equation with forcing term. Appl. Math. Comput. 219, 2732-2738 (2012)

21. Gepreel, KA, Omran, S: Exact solutions for nonlinear partial fractional differential equations. Chin. Phys. B 21, 10204 (2012)

22. Zhang, Y: Formulation and solutions to time fractional generalized Korteweg-de Vries equation via variational methods. Adv. Differ. Equ. 2014, 65 (2014)

23. Benjamin, TB, Bona, JL, Mahony, JJ: Model equations for long waves in nonlinear dispersive system. Philos. Trans. R. Soc. Lond. Ser. A 272, 47-48 (1972)

24. Kolwankar, KM, Gangal, AD: Local fractional Fokker-Planck equation. Phys. Rev. Lett. 80, 214-217 (1998)

25. Chen, W, Sun, HG: Multiscale statistical model of fully-developed turbulence particle accelerations. Mod. Phys. Lett. B 23, 449-452 (2009)

26. Cresson, J: Non-differentiable variational principles. J. Math. Anal. Appl. 307, 48-64 (2005)

27. Jumarie, G: Modified Riemann-Liouville derivative and fractional Taylor series of non-differentiable functions further results. Comput. Math. Appl. 51, 1367-1376 (2006)

28. Jumarie, G: Lagrange characteristic method for solving a class of nonlinear partial differential equations of fractional order. Appl. Math. Lett. 19, 873-880 (2006)

29. Jumarie, G: Fractional partial differential equations and modified Riemann-Liouville derivative new method for solutions. J. Appl. Math. Comput. 24, 31-48 (2007)

30. Wu, GC: A fractional characteristic method for solving fractional partial differential equations. Appl. Math. Lett. 24 1046-1050 (2011)

31. Jumarie, G: New stochastic fractional models for Malthusian growth, the Poissonian birth process and optimal management of populations. Math. Comput. Model. 44, 231-254 (2006)

32. Jumarie, G: Laplace's transform of fractional order via the Mittag-Leffler function and modified Riemann-Liouville derivative. Appl. Math. Lett. 22, 1659-1664 (2009)

33. Almeida, $\mathrm{R}$, Malinowska, $A B$, Torres, $D M$ : A fractional calculus of variations for multiple integrals with application to vibrating string. J. Math. Phys. 51, 033503 (2010)

34. Wu, GC, Lee, EWM: Fractional variational iteration method and its application. Phys. Lett. A 374, 2506-2509 (2010)

35. Malinowska, AB, Sidi Ammi, MR, Torres, DM: Composition functionals in fractional calculus of variations. Commun Fract. Calc. 1, 32-40 (2010)

36. Wu, GC: A fractional Lie group method for anonymous diffusion equations. Commun. Fract. Calc. 1, 23-27 (2010)

37. Ebaid, A, Aly, EH: Exact solutions for the transformed reduced Ostrovsky equation via the F-expansion method in terms of Weierstrass-elliptic and Jacobian-elliptic functions. Wave Motion 49, 296-308 (2012)

38. Hong, B, Lu, D: New exact Jacobi elliptic function solutions for the coupled Schrodinger-Boussinesq equations. J. Appl. Math. 2013, Article ID 170835 (2013)

10.1186/1687-1847-2014-286

Cite this article as: Gepreel: Explicit Jacobi elliptic exact solutions for nonlinear partial fractional differential equations. Advances in Difference Equations 2014, 2014:286

\section{Submit your manuscript to a SpringerOpen ${ }^{\circ}$ journal and benefit from:}

- Convenient online submission

Rigorous peer review

- Immediate publication on acceptance

- Open access: articles freely available online

- High visibility within the field

- Retaining the copyright to your article 